\title{
Normal Mode Relaxation of Polyisoprene in Blends with Vinyl Polybutadienes
}

\author{
M. J . Schroeder \\ Department of Chemistry, United States Naval Academy, Annapolis, Maryland 21402
}

\section{M. Roland*}

Chemistry Division, Code 6120, Naval Research Laboratory, Washington, D.C. 20375-5342

Received August 11, 1998; Revised Manuscript Received J anuary 19, 1999

\begin{abstract}
When probe chains are dispersed in a network or higher molecular weight matrix, the expectation from the reptation model is that the terminal relaxation function will narrow, due to suppression of constraint release. Previous experiments found no support for this prediction, although they relied on time-temperature superpositioning to obtain spectra. These studies also made use of polyisoprene (PI)/1,4-polybutadiene blends, whose thermodynamic miscibility is problematic.We report herein measurements acquired with a broad-band dielectric spectrometer of the terminal relaxation of $\mathrm{PI}$ in blends with polybutadiene having substantial 1,2-content. This mixture is thermodynamically miscible, thus excluding the possibility of phase separation. Corroborating earlier work, we find that the experimental behavior is qualitatively opposite to the prediction. Suppression of constraint release broadens the terminal dispersion, increasing the deviation from the theoretical relaxation function. This suggests that the common assumption that reptation and constraint release are independent processes is incorrect.
\end{abstract}

\section{Introduction}

The terminal relaxation function for pure reptation,

$$
\mathrm{G}_{\text {rep }}(\mathrm{t})=\mathrm{G}_{\mathrm{N}}^{0} \sum_{\mathrm{p} ; \mathrm{odd}} \frac{8}{\pi^{2} \mathrm{p}^{2}} \exp \left(-\frac{\mathrm{p}^{2} \mathrm{t}}{\tau_{\text {rep }}}\right)
$$

where $\mathrm{G}_{\mathrm{N}}^{0}$ is the plateau modulus and $\tau_{\text {rep }}$ the reptation time, is narrower than the experimental spectrum for monodisperse polymers. ${ }^{1}$ Since the tube model considers only a single chain constrained by entanglements, the deviation from experiment presumably reflects motion of the entanglement constraints themselves. ${ }^{1-6}$ By assuming that this "constraint release" process transpires independently of chain reptation, the relaxation modulus can be expressed as a simple product ${ }^{2,7,8}$

$$
\mathrm{G}(\mathrm{t})=\mathrm{G}_{\mathrm{rep}}(\mathrm{t}) \mathrm{G}_{\mathrm{cr}}(\mathrm{t})
$$

where $G_{c r}(t)$ is the rate of stress relaxation due to constraint release. The additional relaxation reduces the terminal relaxation time, according to

$$
\tau^{-1}=\tau_{\text {rep }}^{-1}+\tau_{\text {cr }}^{-1}
$$

where $\tau_{\mathrm{cr}}$ is the time scal e for constraint release; it also broadens the terminal dispersion, yiel ding better agreement with experimental results.

As pointed out by Graessley, ${ }^{7}$ for probe chains dissolved in a higher molecular weight matrix, the contribution from constraint release should be quite small. Even though different models describe the effect of constraint release somewhat differently, they have in common (with one exception ${ }^{6}$ ) the assumption that it transpires independently of reptation itself. Thus, the prediction that suppression of constraint release will yield a relaxation function having the form of eq 1 is a basic result of the reptation approach.
Mechanical measurements on networks containing unattached chains, in which constraint release is suppressed due to the cross-linking, indeed show the relaxation of probe chains to be retarded, consistent with eq 3. ${ }^{9-12}$ Unfortunately, mechanical data on blends are difficult to analyze, since the response of the matrix overwhelms that of the probe chains.

A variety of other experimental methods have been brought to bear on the problem of resolving the component dynamics in blends. These include deuterium NMR, ${ }^{13-16}$ infrared dichroism, ${ }^{17-20}$ and rheoptical techniques utilizing birefringence and dichroism. ${ }^{21-23}$ The most interesting result of these studies is the discovery of orientational ("nematic") coupling, indicating that the terminal relaxation is to some degree cooperative. Such behavior is at odds with the factorization expressed by eq 2.

A very useful technique for resolving the component dynamics is dielectric spectroscopy. For example, segmental relaxation in blends of polyisoprene and polybutadiene (PBD) has been measured over a range of temperatures and compositions using combined dielectric and mechanical spectroscopies. ${ }^{24}$ The results were in good agreement with other techniques. ${ }^{25}$ For the study of terminal relaxation, polyisoprene is of particular interest because it has a dipole moment parallel to the chain contour, whereby its normal mode is dielectrically active. ${ }^{26}$ This means that in blends with polybutadiene only the PI contributes at low frequencies, enabling motion of its end-to-end chain vector to be obtained without interference from the other component.

Dielectric measurement of PI detects the orientational memory of the chains, which differs from the viscoelastic relaxation reflecting decay of orientational anisotropy. 27 However, assuming independence of reptation and constraint release, the dielectric relaxation function is also given by eq 2, with $\mathrm{G}_{\mathrm{rep}}(\mathrm{t})$ and $\mathrm{G}_{\mathrm{cr}}(\mathrm{t})$ replaced by 
Table 1. Polymers

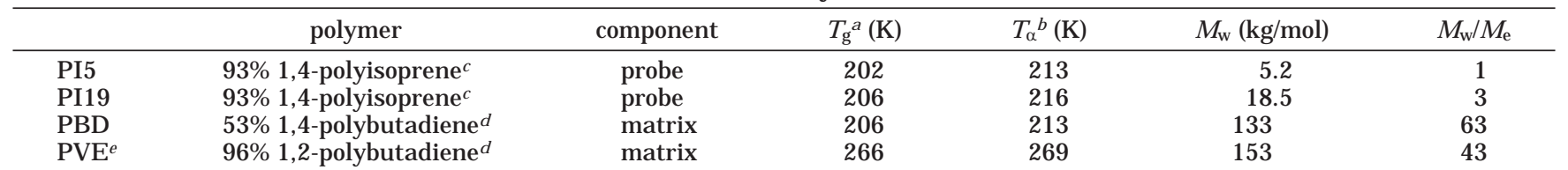

${ }^{a}$ DSC cooling at $10 \mathrm{deg} / \mathrm{min} .{ }^{\mathrm{b}}$ Temperature at which segmental relaxation time measured dielectrically equals $1 \mathrm{~s} .{ }^{\mathrm{c}} \mathrm{Goodyear}$ Tire $\&$ Rubber Co. ${ }^{d}$ Bridgestone/Firestone Inc. e Poly(vinylethylene).

the corresponding diel ectric functions for pure reptation and constraint release, respectively. This factorized form predicts narrowing of the dielectric spectrum on suppression of constraint release.

Adachi et al. have carried out a series of dielectric investigations of PI blended with 1,4-polybutadiene, the latter either cross-linked or substantially higher in molecular weight than the PI. Blending increased the normal mode relaxation time of the PI, consistent with a diminished contribution from constraint release (eq 3). ${ }^{28}$ Master curves of PI's normal mode spectrum were broader when dispersed in networks of 1,4-PBD than the reptation prediction (eq 1 ), although resort had to be made to time-temperature superpositioning due to the limited frequency range of the dielectric spectrometer. ${ }^{29}$ Similar results have been obtained for PI dispersed in linear 1,4-PBD. When the latter is wellentangled, master curves for the PI normal mode peak were invariably broader than eq $1.30-33$

It is a provocative finding that, even in the absence of constraint release, experimental relaxation functions still deviate from the reptation model. At least two possible limitations of the existing studies are apparent. A reliance on time-temperature superpositioning introduces some uncertainty into any comparison of peak shapes. Moreover, as Adachi and Kotaka have pointed out $^{34}$ the possibility exists for phase separation in PI blended with 1,4-PBD. If some segregation occurs, the entangl ements of the PI will relax more rapidly than if the local environment of these probe chains were purely 1,4-PBD.

The purpose of the work described herein was to provide corroboration of existing results by addressing these possible shortcomings. Normal mode spectra of dilute PI in blends were obtained over a broad range of measured frequencies, obviating any need for timetemperature superpositioning. The matrix polymer was PBD having substantial vinyl content (i.e., 1,2-polybutadiene microstructure). It is well-established that miscibility with PI increases with vinyl content of the PBD component. ${ }^{35}$ The blend even exhibits a negative interaction parameter when the 1,2-content is more than $90 \% .36,37$

\section{Experimental Section}

The polybutadienes were prepared by J .E. Hall of Bridgestone/Firestone Inc. and the polyisoprenes by A.F. Halasa of the Goodyear Tire \& Rubber Co. Syntheses were by anionic polymerization using butyllithium initiators. Molecular weights are listed in Table 1, along with the average number of entangl ements per chain. For PBD, this was estimated from $\mathrm{M}_{\mathrm{e}}$ values reported for polybutadienes of comparable chemical structure. 38,39 For 1,4-polyisoprene, the entangl ement molecular weight is in the range from 4300 to $6200 \mathrm{~g} / \mathrm{mol}^{2,34,40-43}$ although in blends with polybutadiene, $M_{e}$ is somewhat reduced. $29,44,45$ The polydispersity of the polyisoprene was 1.06, while the polybutadienes had broader molecular weight distributions, $\mathrm{M}_{\mathrm{w}} / \mathrm{M}_{\mathrm{n}}=1.14$ and 1.68 for the PVE and the PBD, respectively.
The blends contained $15 \mathrm{wt} \%$ of the PI, the lowest concentration at which dielectric measurements could be reliably obtained. Experiments on samples with a higher PI content yielded results qualitatively the same to those reported herein. Homogeneous blends of the PI in either polybutadiene were prepared by solvent-casting 5\% cyclohexane solutions. The solvent was allowed to evaporate slowly, yielding transparent films, which were then vacuum-dried for at least $24 \mathrm{~h}$ at room temperature prior to measurements. The polybutadienes were not cross-linked, to avoid possible complications with the use of networks in this type of study. ${ }^{46}$

I sothermal dielectric measurements were obtained using a time domain spectrometer (Imass Inc.) having a frequency range from $10^{-4}$ to $10^{4} \mathrm{~Hz}$. A parallel plate geometry was employed, with a guard ring on the detector side. The sample dimensions were typically $0.2 \mathrm{~mm}$ thick and $25 \mathrm{~mm}$ in diameter. For measurements on the neat PI5 only, a liquid cell (HP16452A) was used due to the polymer's low viscosity. A Delta Design model 9023 liquid-nitrogen-cooled oven provided temperature control to better than $\pm 0.2 \mathrm{~K}$. Samples were allowed to equilibrate for at least a $1 \mathrm{~h}$ after attaining the desired temperature.

\section{Results and Discussion}

From the respective values for the number of entanglements per chain (Table 1), the polybutadienes used as the matrix polymer are expected to relax much more slowly than the PI. We quantify this from the terminal relaxation behavior, measured mechanically since the dielectric normal mode for PBD has negligible intensity. Comparisons of mechanical and dielectric relaxation for these polymers have been published. 24,27,40,47-49

Figure 1 displays the frequency in the terminal zone at which the storage and loss moduli are equal. Representative storage and loss moduli spectra for PI 19 are shown as an inset. We omit data for PI5, which has a negligible plateau due to its low $\mathrm{M}_{\mathrm{w}} / \mathrm{M}_{\mathrm{e}}$ (Table 1 ); however, its terminal relaxation is almost 2 orders of magnitude faster than for PI19. Thus, relaxation of either PI will transpire on a time scale too short for entanglements to be released via motion of the polybutadienes. This absence of constraint release leads to the expectation that eq 1 should describe the rel axation function of the PI probe chains.

The normal mode spectra were measured for the neat $\mathrm{PI}$ and the blends. Displayed in Figure 2 are normal mode relaxation times, defined as the reciprocal of the frequency of the maximum in the dielectric loss peak. This "most probable" relaxation time is reported to be insensitive to polydispersity. ${ }^{50}$ Dissol ving the PI into the highly entangled matrix polymers significantly retards its relaxation, consistent with Figure 1 and eqs 2 and 3.

An unresolved issue in polymer rheology concerns the molecular weight dependence of the terminal relaxation time. For reptation without constraint release, a cubic power dependence on molecular weight is expected. ${ }^{1-3,7,38,46}$ Previous results on PI dispersed in high molecular matrices and networks have indicated the 


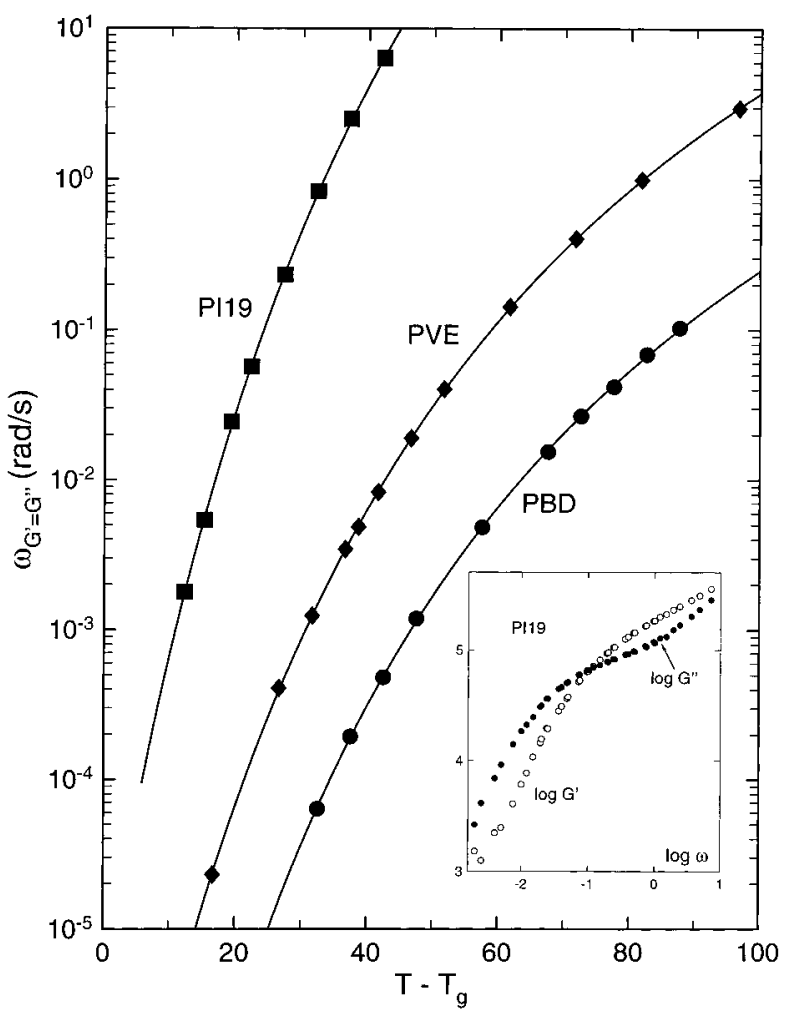

Figure 1. Frequency in the terminal zone of the mechanical spectrum at which the storage and loss moduli are equal for the neat polybutadienes ( $\bullet$ and $\bullet$ ) and for the higher molecular weight PI (ם). The data illustrate that in the blends matrix relaxation will be much slower than relaxation of the probe polymer. The abscissa, drawn from free vol ume ideas, ${ }^{38}$ is an approximate correction for differences in $\mathrm{T}_{\mathrm{g}}$ among the polymers. Shown as an inset are representative storage and loss moduli spectra for the PI 19.

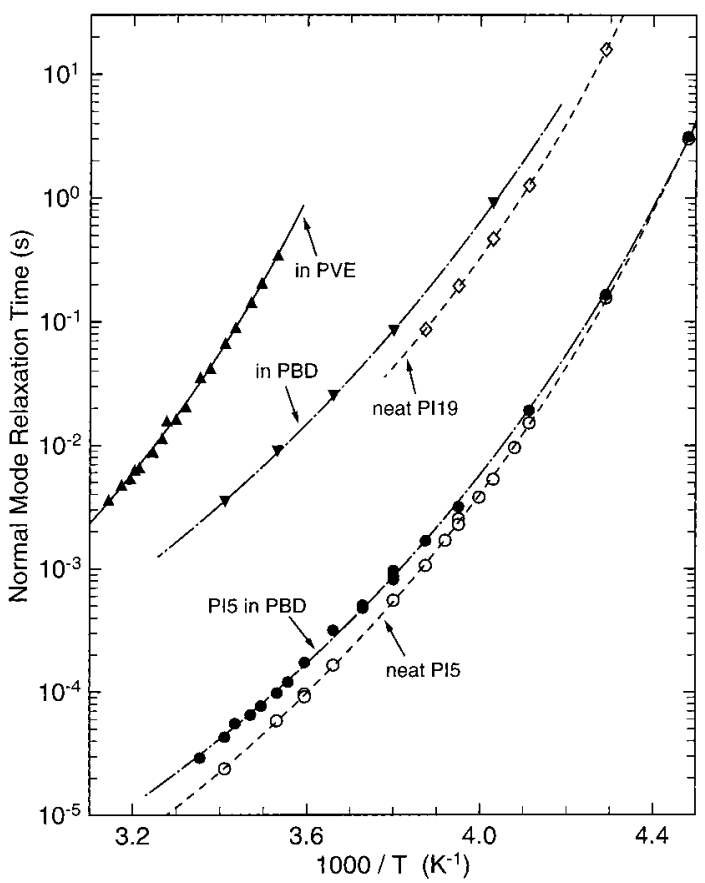

Figure 2. Normal mode relaxation time of the polyisoprenes measured neat (hollow symbols) and in blends (sol id symbols) with polybutadiene.

exponent to be 3 , which is consistent with theory, or $3.6,29,30,34$ which is not. This power-law exponent cannot be assessed herein, since we have only two data points. We mention in passing that the ratio of the normal mode

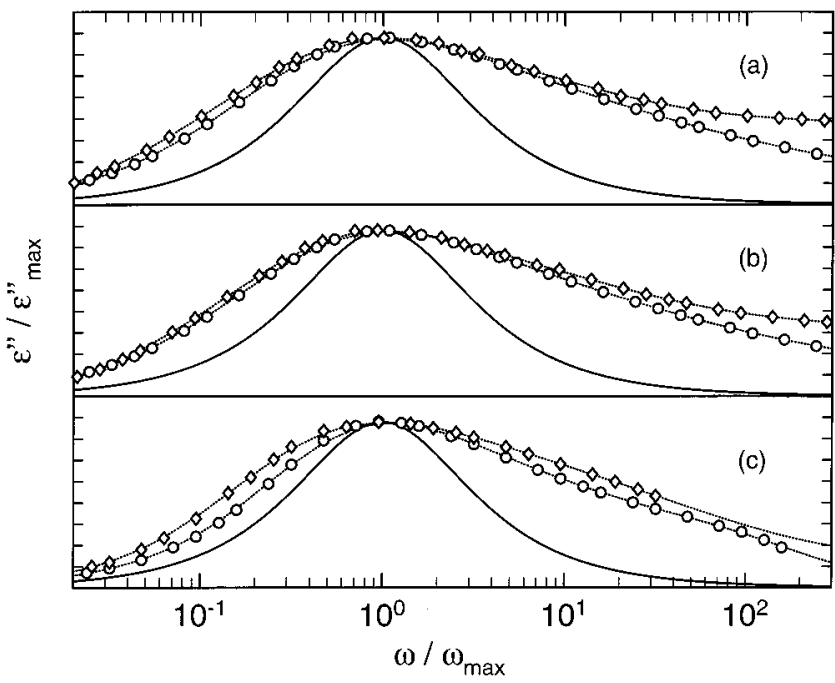

Figure 3. Normal mode dispersion measured for PI neat $(O)$ and in $15 \%$ blends with polybutadiene $(\diamond)$ : (a) PI 19 in PVE, (b) PI 19 in PBD, (c) PI5 in PBD. The data have been shifted to superimpose the peaks. When diluted with higher molecular weight polymer, the terminal relaxation of the $\mathrm{PI}$ is always broader. All curves are substantially broader than the reptation prediction in the absence of constraint release (eq 1, solid line).

relaxation times for the PIs in Figure 2 corresponds to a 3.5 power dependence on molecular weight.

In Figure 3 are shown the normal mode dispersions measured for PI neat (circles) and in blends (diamonds). These curves, which have been shifted to allow comparison of the peak breadths, were all obtained at a single temperature; that is, the data were not timetemperature superpositioned. Also shown in the figure is eq 1 (solid line), the reptation prediction in the absence of constraint release. Clearly the latter is significantly narrower than the experimental dispersions. Moreover, for both PIs, and for either matrix, there is broadening of the relaxation function with blending. The effect of suppression of constraint release is thus qualitatively opposite to the prediction of eq 2 .

\section{Summary}

The result that probe chains dispersed in a higher molecular weight matrix have their relaxation slowed with concomitant broadening of the relaxation function corroborates previously findings. ${ }^{28-33}$ Moreover, possible criticisms of the earlier work (i.e., reliance on master curves and the possibility of phase separation in blends of PI with 1,4-PBD) are moot herein. Thus, it is firmly established that there is a qualitative discrepancy between theories predicting that suppression of constraint release narrows the relaxation function and experiments, which show broadening.

Since the matrix polybutadiene has negligible dipole moment parallel to the chain contour, it is not oriented by the applied electric field. Accordingly, the nematic coupling effect observed in blends, whereby short chains retain some orientation until the whole system relaxes, ${ }^{51-53}$ is not operative in the present experiments. On the other hand, Watanabe et al. ${ }^{27,33}$ have suggested that the matrix's influence on terminal chain behavior extends beyond the effect of entanglements addressed by the reptation model. Diel ectric studies of probes with inverted dipoles $31,54,55$ have indicated the existence of some unspecified coupling in the dynamics of the blend components. This idea merits further exploration, par- 
ticularly in light of the fact that the model of Watanabe and Tirrell ${ }^{6}$ does not assume constraint release to be independent of probe conformation. It is this assumption which yields the factorized form of eq 2 . However, this model 6 predicts that the lower frequency terminal dynamics will be independent of the rate of tube renewal, a result at odds with the broadening seen in Figure 3.

Finally, we point out an alternative approach to the low-frequency dynamics of polymers that is diametrically opposite to the single chain theory of reptation. The coupling model, based on general principles of constraint dynamics in complex fluids, interprets broadening of the relaxation function to be a direct consequence of enhanced intermol ecular cooperativity. ${ }^{56}$ The data in Figure 3 are consistent with this idea, insofar as the absence of constraint release increases the severity of the entanglement constraints. ${ }^{57-59}$ However, the coupling model makes a general prediction that broader relaxation functions are associated with relaxation times which are more sensitive to temperature. This appears to be contrary to the results in Figure 2, which indicate a somewhat reduced dependence on temperature for PI's normal mode in the blend relative to the neat polymer.

Acknowledgment. The authors thank J .E. Hall of Bridgestone/F irestone Inc. and A.F. Halasa of the Goodyear Tire \& Rubber Co. for kindly providing the polybutadienes and polyisoprenes, respectively. Stimulating discussions with K. L. Ngai are appreciated. This work was supported by the Office of Naval Research and M.J .S. through the Naval Academy Research Council (ONR Grant N0001496WR20008).

\section{References and Notes}

(1) Doi, M.; Edwards, S. F. The Theory of Polymer Dynamics; Clarendon: Oxford, 1986.

(2) Pearson, D. S. Rubber Chem. Technol. 1987, 60, 439.

(3) Daoud, M.; de Gennes, P. G. J . Polym. Sci., Polym. Phys. Ed. 1979, 17, 1971.

(4) Marucci, G. J . Polym. Sci., Polym Phys. Ed. 1985, 23, 159.

(5) Rubinstein, M.; Helfand, E.; Pearson, D. S. G. Macromol ecules $1987,20,822$.

(6) Watanabe, H.; Tirrell, M. Macromolecules 1989, 22, 927.

(7) Graessley, W. W. Adv. Polym. Sci. 1982 47, 68.

(8) Rubinstein, M.; Colby, R. H. J . Chem. Phys. 1988, 89, 5291.

(9) Nelb, G. W.; Pedersen, S.; Taylor, C. R.; Ferry, J . D. J . Polym. Sci., Polym. Phys. Ed. 1980, 18, 645.

(10) Granick, S.; Pedersen, S.; N elb, G. W.; Ferry, J . D. J . Polym. Sci., Polym. Phys. Ed. 1981, 19, 1745.

(11) Poh, B. T.; Adachi, K.; Kotaka, T. Macromolecules 1987, 20 , 2569.

(12) Ndoni, S.; Vorup, A.; Kramer, O. Macromolecules 1998, 31, 3353.

(13) Deloche, B.; Samulski, E. T. Macromolecules 1981, 14, 575.

(14) J acobi, M. M.; Stadler, R.; Gronski, W. Macromol ecules 1986, $27,964$.

(15) Sotta, P.; Deloche, B. Macromolecules 1987, 20, 2769.

(16) Chung, G.-C.; Kornfield, J . A.; Smith, S. D. Macromolecules 1994, 27, 964.

(17) Faivre, J. P.; J asse, B.; Monnerie, L. Polymer 1985, 26, 879.

(18) Saito, H.; Takahashi, M.; Inoue, T. J . Polym. Sci., Polym. Phys. Ed. 1988, 26, 1761.
(19) Tassin, J .-F.; Baschwitz, A.; Moise, J .-Y.; Monnerie, L. Macromolecules 1990, 23, 1879.

(20) Zemel, I. S.; Roland, C. M. Polymer 1992, 33, 4522.

(21) Kornfield, J . A.; Fuller, G. G.; Pearson, D. S. Macromolecules 1989, 22, 1334

(22) Kornfield, J . A.; Fuller, G. G.; Pearson, D. S. Macromolecules 1991, 24, 5429.

(23) Zawada, J .; Fuller, G. G.; Colby, R. H.; Fetters, L. J .; Roovers, J. Macromol ecules 1994, 27, 6861.

(24) Alegria, A.; Colmenero, J.; Ngai, K. L.; Roland, C. M. Macromolecules 1994, 27, 4486.

(25) Ngai, K. L.; Roland, C. M. Macromolecules 1995, 28, 4033.

(26) Stockmayer, W. H. Pure Appl. Chem. 1967, 15, 539.

(27) Watanabe, H.; Yao, M.-L.; Osaki, K. Macromolecules 1996, 29, 97.

(28) Adachi, K.; Kotaka, T. Prog. Polym. Sci. 1993, 18, 585.

(29) Adachi, K.; Nakamoto, T.; Kotaka, T. Macromolecules 1989, $22,3111$.

(30) Adachi, K.; Wada, T.; Kawamoto, T.; Kotaka, T. Macromolecules 1995, 28, 3588.

(31) Watanabe, H.; Yamazaki, M.; Yoshida, H.; Adachi, K.; Kotaka, T. Macromolecules 1991, 24, 5365.

(32) Poh, B. T.; Adachi, K.; Kotaka, T. Macromolecules 1996, 29, 6317.

(33) Watanabe, H.; Urakaw, O.; Yamada, H.; Yao, M.-L. Macromolecules 1996, 29, 755

(34) Adachi, K.; Kotaka, T. J . Non-Cryst. Solids 1991, 131-133, 723.

(35) Trask, C. A.; Roland, C. M. Polym. Commun. 1988, 29, 332.

(36) Roland, C. M. Macromolecules 1987, 20, 2557.

(37) Tomlin, D. W.; Roland, C. M. Macromolecules 1992, 25, 2994.

(38) Ferry, J . D. Viscoelastic Properties of Polymers; Wiley: New York, 1980; Chapter 10.

(39) Fetters, L. J .; Lohse, D. J .; Colby, R. H. In Physical Properties of Polymers Handbook; Mark, J . E., Ed.; American Institute Physics Press: Woodbury, NY, 1996; Chapter 24.

(40) Santangelo, P. G.; Roland, C. M. Macromolecules 1998, 31, 3715.

(41) Roland, C. M. Macromolecules 1992, 25, 7031.

(42) Gotro, J. T.; Graessley, W. W. Macromol ecules 1984, 17, 2767.

(43) Graessley, W. W.; Edwards, S. F. Polymer 1981, 22, 1329.

(44) Roovers, J .; Toporowski, P. M. Macromolecules 1991, 25, 1096.

(45) Arendt, B. H.; Kannan, R. M.; Zewail, M.; Kornfield, J . A.; Smith, S. D. Rheol. Acta 1994, 33, 322.

(46) Lodge, T. P.; Rotstein, N. A.; Prager, S. Adv. Chem. Phys. 1990, 79, 1.

(47) Adachi, K.; Kotaka, T. Macromolecules 1984, 17, 120.

(48) Adachi, K.; Yoshida, H.; Fukui, F.; Kotaka, T. Macromol ecules 1990, 23, 3138.

(49) Colmenero, J .; Alegria, A.; Santangelo, P. G.; Ngai, K. L.; Roland, C. M. Macromolecules 1994, 27, 407.

(50) Imanishi, Y.; Adachi, K.; Kotaka, T. J . Chem. Phys. 1988 89, 7585.

(51) Doi, M.; Pearson, D.; Kornfield, J .; Fuller, G. Macromolecules 1989, 22, 1488.

(52) Watanabe, H.; Kotaka, T.; Tirrell, M. Macromolecules 1991 , 24, 201.

(53) Doi, M.; Watanabe, H. Macromolecules 1991, 24, 744.

(54) Watanabe, H.; Yamazaki, M.; Yoshida, H.; Kotaka, T. Macromolecules 1991, 24, 5372.

(55) Watanabe, H.; Urakawa, O.; Kotaka, T. Macromol ecules 1994 $27,3525$.

(56) Ngai, K. L.; Plazek, D. J . Rubber Chem. Technol. 1995, 68, 376.

(57) Ngai, K. L.; Plazek, D. J .; Rendell, R. W. Rheol. Acta 1997 $36,307$.

(58) Ngai, K. L.; Roland, C. M. J . Polym. Sci., Polym. Phys. Ed. 1997, 35, 2503.

(59) Santangelo, P. G.; Ngai, K. L.; Roland, C. M. Polymer 1998, 39,681 .

MA981271L 